\title{
Photonic Remote Microwave Frequency \\ Measurement With a Tunable Measurement Range Based on Dispersion Compensation Technology
}

\section{Qingqing Meng}

Air Force Engineering University https://orcid.org/0000-0002-9528-9163

\section{Zihang Zhu}

Air Force Engineering University

Tao Lin

Air Force Engineering University

He Li

Air Force Engineering University

\section{Guodong Wang}

Air Force Engineering University

\section{Lanfeng Huang}

Air Force Engineering University

\section{Xuan Li}

Air Force Engineering University

\section{Longqiang Yu}

Air Force Engineering University

Shanghong Zhao ( $\sim$ zhaoshangh@aliyun.com )

Air Force Engineering University

\section{Research Article}

Keywords: remote frequency measurement, microwave photonic, dispersion compensating fiber, dualpolarization dual-drive Mach-Zehnder modulator (DPol-DMZM)

Posted Date: December 15th, 2021

DOI: https://doi.org/10.21203/rs.3.rs-1069251/v1

License: (c) (1) This work is licensed under a Creative Commons Attribution 4.0 International License.

Read Full License 


\title{
Photonic remote microwave frequency measurement with a tunable
}

\section{measurement range based on dispersion compensation technology}

\author{
Qingqing Meng, Zihang Zhu, Tao Lin, He Li, Guodong Wang, Lanfeng Huang, Xuan Li, Longqiang Yu, \\ and Shanghong Zhao* \\ Air Force Engineering University, College of Information and Navigation, Xi'an 710077, China \\ *Corresponding author: zhaoshangh@aliyun.com
}

\begin{abstract}
In this paper, a novel and efficient photonic-assisted remote frequency measurement (RFM) system with a significantly simplified structure and flexible operation range is proposed. By simply changing the dispersion coefficient or length of the dispersion medium in the central station (CS), the microwave frequency measurement range in the remote antenna unit (RAU) can be tuned. In this system, the RAU and the CS is separated to ensure the concealment and safety of the signal processing unit. The measurement range of the RFM system can be tuned easily during the measurement process without system reconstruction, and different RAUs located at different places can be controlled to work at the same measurement range. The simulation results show that a frequency measurement over the high frequency range $(>18$ $\mathrm{GHz}$ ) can be achieved with a measurement error better than $\pm 0.2 \mathrm{GHz}$. Noteworthy, the impact of the non-ideal factors such as bias drift, intensity noise, phase noise, the equivalent deviation of the polarization beam splitter (PBS), and the dispersion value of the single mode fiber (SMF) is also discussed. It has been proved that they have little influence on the system performance over the high frequency range.
\end{abstract}

Key words: remote frequency measurement; microwave photonic; dispersion compensating fiber; dual-polarization dual-drive Mach-Zehnder modulator (DPol-DMZM).

\section{Introduction}

Microwave frequency measurement plays an important role in electronic warfare, radar warning receivers, and wireless communication systems [1,2]. These applications require accurate measurement of the frequency information of unknown intercepted microwave signals before further signal analysis and processing. However, the radio frequency (RF) receivers are usually exposed to the detection beams of the enemy to directly intercept the related signal in a complex electromagnetic environment. In fact, electrical-based frequency measurement technologies have been well developed. However, due to electrical bottleneck, the measurement range of the conventional electrical frequency measurement approach is usually restricted to $18 \mathrm{GHz}[3,4]$. Moreover, they are vulnerable to the electromagnetic interference(EMI). Photonic-assisted frequency measurement provides a promising solution to these issues due to the inherent advantages of low loss, large bandwidth, small size, and immunity to EMI. 
In general, to measure the frequency, a relationship between the microwave frequency and some specific parameters of the microwave photonic subsystem should be established, such as frequency-to-space mapping [5,6], frequency-to-time mapping [7,8], and frequency-to-power mapping [9-13]. It should be mentioned that these methods seldom considered the security of the receivers that are expensive and full-technology equipped. An efficient way is to separate the cheap antenna unit and the expensive signal processing unit far away from each other through a cable or a single mode fiber (SMF). Thus the signal processing unit can be hidden in a well-protected place. Fortunately, a novel photonic-assisted frequency measurement method based on frequency-to-power mapping utilizing a few-mode fiber that could realize remote microwave frequency measurement is proposed [14]. However, the frequency measurement range is limit and not tunable. Especially, for different RAUs locate in different places, the operation range is also varied. Moreover, two lasers with different wavelengths are employed, which increases the system complexity.

To cope with future reconnaissance and improve the concealment of the signal processing unit, the RF receiving unit and the signal processing unit should be separated and far away from each other. In another word, remote microwave signal frequency measurement is required. In this case, several significant problems should be raised: 1) how to provide an optical carrier to the RAU, 2) how to make frequency measurement system more flexible to suitable for arbitrary RAU locations, 3) how to endow the fixed RAU with flexible frequency measurement range. In this paper, a remote frequency measurement (RFM) method with significantly simplified structure and tunable operation range is proposed. Only one dual-polarization dual-drive Mach-Zehnder modulator (DPol-DMZM) is employed in this system. Noteworthy, the DPol-DMZM is settled in a remote station to receive RF signals and introduce them into the optical field. Then, transfer the modulated optical signal to the signal processing unit in the central station (CS) by SMF to extract the frequency information. In this structure, an amplitude comparison function (ACF) is constructed to avoid unpredictable RF power fluctuation, so that the system response can be more stable. It should be noted that in the $\mathrm{CS}$, a dispersion medium such as dispersion compensated fiber (DCF) is adopted to flexibly control the frequency measurement range. By changing the dispersion coefficient or length of the dispersion medium in the CS, the measurement range of the frequency measurement system can be easily tuned during the measurement process without system reconstruction. Thus, the remote detecting range can be designed in the CS to make it suitable for different RAUs that locate at different places, which enhances the performance of the system. Discussions on the tunability of frequency measurement, the impact of non-ideal factors namely bias drift, intensity noise, phase noise, the equivalent deviation of PBS and the dispersion value of the SMF are considered.

\section{Principle}

The overall schematic diagram of the proposed photonic-assisted remote microwave frequency measurement system is shown in Fig. 1. In Fig. 1(a), the whole system is divided into two parts, remote antenna unit (RAU) and central station (CS). The RAU is located at remote place to directly receive the unknown RF signal and introduce it into the optical field. Then, the modulated optical signal is sent back to the CS through single mode fiber (SMF). In general, to guarantee the transmitting quality, two-fiber system is widely considered [15]. In 
two-fiber system, the downlink fiber and the uplink fiber are the two physically isolated fibers. Usually, to reduce the cost, the optical source of the RAU is provided by the CS. Here, a laser diode (LD) is set in CS. It provides a high quality optical carrier and sent it to the RAU through the downlink SMF. In the RAU, the received RF signal is modulated into the optical field by a modulator. Differently, the modulated optical signal traverses an uplink SMF and arrives at the CS to perform specific signal processing to extract signal frequency.

Fig. 1(b) shows the detail structure of the RFM system. It mainly consists of an LD, two polarization controllers (PC1, and $\mathrm{PC} 2$ ), two optical circulators (OC1, and $\mathrm{OC} 2$ ), a dual-polarization dual-drive Mach-Zehnder modulator (DPol-DMZM), single mode fiber (SMF), a polarization beam splitter (PBS), and two photodetectors (PDx, and PDy). Considering the remote detecting requirement, the RAU should be as concise as possible. Therefore, only the DPol-DMZM, OC2 and a PC2 are located in the RAU, the rest of these devices are all settled in the CS.

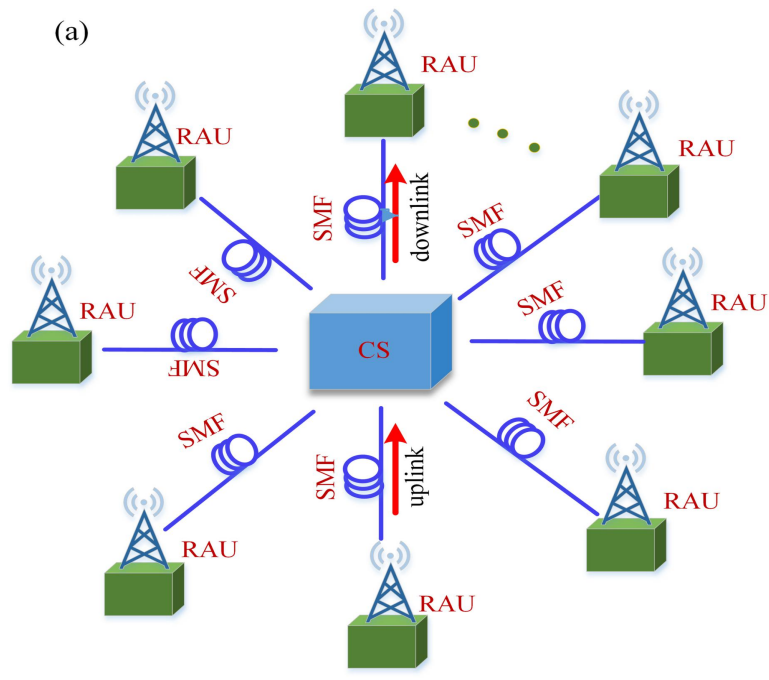

(b)
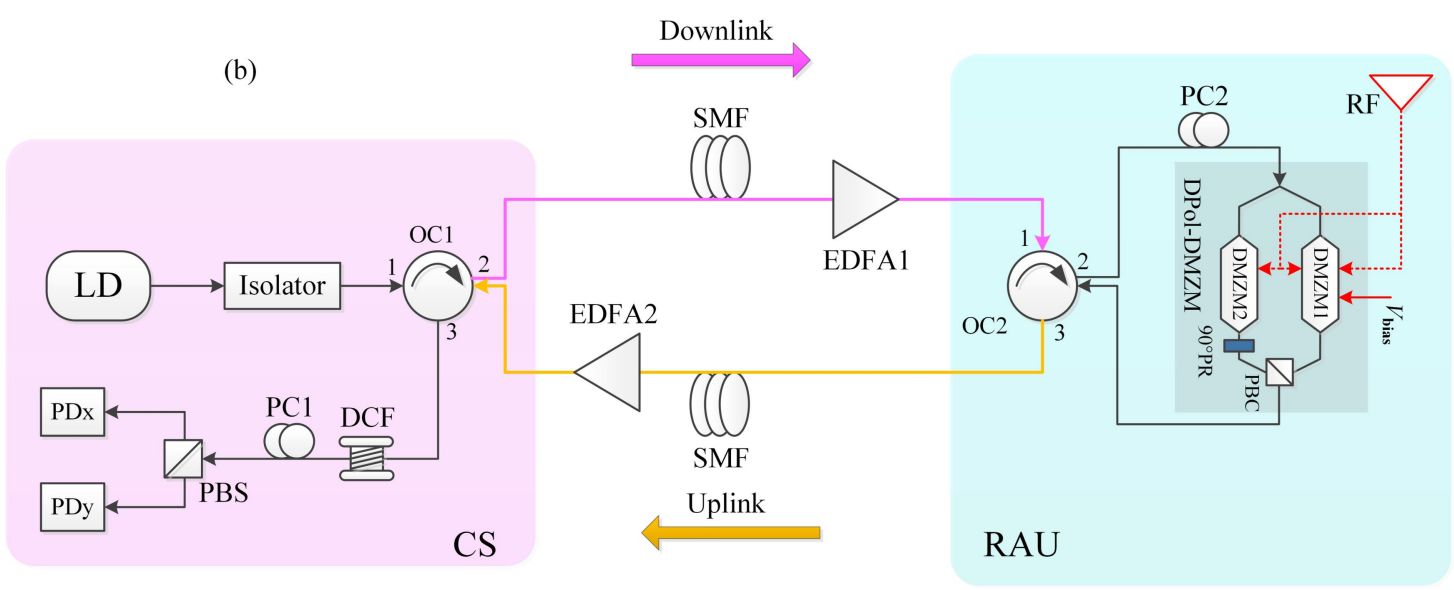

Fig. 1. (a) The general remote detection structure diagram. (b) The detail construction of the proposed photonic-assisted remote frequency measurement system.

As Fig. 1(b) shows, to achieve remote microwave frequency measurement, a continuous-wave light from the CS is injected into port1 of the $\mathrm{OC} 1$ and outputs from the port2 that connects to the downlink SMF. The optical carrier is led to the RAU through the downlink SMF. In the RAU, the optical carrier from the CS gets into the port 1 of OC2 and is guided to 
port2. The guided carrier is then sent to a DPol-DMZM, which is employed to carry the received unknown RF signal from the antenna. It is an integrated modulator with two dual-drive Mach-Zehnder modulators (DMZM1, and DMZM2) embedded in two arms. Moreover, due to the followed $90^{\circ} \mathrm{PR}$ in the lower arm, this modulator is a polarization-multiplexed device. The optical carrier is first oriented to align to one principal axes of the DPol-DMZM by PC2. Then, in the modulator, it is first split into two branches that are equal in power. In the upper branch, the unknown RF signal is equally split and applied to two RF ports of the DMZM1. The DC voltage is set to quadrature transmission point (QTP) to generate a double sideband (DSB) signal. In the lower branch, the RF signal is only applied to one arm of the DMZM2 while another is null, so that the phase-modulated signal can be generated. After $90^{\circ} \mathrm{PR}$, the two modulated optical signals in the two branches are polarization orthogonal and finally recombined by a PBC.

Supposing that the optical carrier from the LD is given by $E_{\text {in }}(t)=E_{0} \exp \left(\mathrm{j} \omega_{c} t\right)$, the RF signal to be measured is $V_{R F}(t)=V \sin \left(\omega_{R F} t\right)$, where $E_{0}$ and $\omega_{\mathrm{c}}$ are the amplitude and the angular frequency of the optical carrier, respectively. $V$ and $\omega_{R F}$ are the amplitude and the angular frequency of the unknown microwave signal, respectively. Under small-signal condition, the sidebands higher than first-order can be ignored, if the signal power is low. Therefore, the output signal of the DPol-DMZM along the two orthogonal directions can be expressed as

$$
\left[\begin{array}{c}
E_{X} \\
E_{Y}
\end{array}\right]=\frac{\sqrt{2}}{4} E_{i n}\left\{\begin{array}{l}
{\left[(j+1) J_{0}(m)+(j-1) J_{1}(m) \exp \left(j \omega_{\mathrm{RF}} t\right)-(j-1) J_{1}(m) \exp \left(-j \omega_{\mathrm{RF}} t\right)\right]} \\
{\left[J_{0}(m)+J_{1}(m) \exp \left(j \omega_{\mathrm{RF}} t\right)-J_{1}(m) \exp \left(-j \omega_{\mathrm{RF}} t\right)+1\right]}
\end{array}\right\}
$$

Where $m=\pi V_{\mathrm{RF}} / \sqrt{2} V_{\pi}$ is the modulation index, $V_{\pi}$ denotes half-wave voltage of the DMZM1.

$J_{\mathrm{n}}($.$) is the n$ th-order Bessel function of the first kind. As can be seen from Eq. (1), the signal includes three different frequency components in the form of DSB and PM. The output orthogonal signals are combined by a polarization beam combiner (PBC) and transmitted back to the CS. It goes from port 2 to port 3 of the OC2 and traverses through the uplink SMF. In the $\mathrm{CS}$, the received optical signal is guided from port 2 to port 3 of the OC1. Noteworthy, the guided optical signal is then sent to a dispersion medium. Here, we choose a DCF as the example. According to the transfer function of a dispersive fiber link [16], the dispersion-induced phase shifts of the \pm 1 st order sidebands are designated as $\varphi_{1}, \varphi_{2}$. The optical expression can be written as [17]

$$
\left[\begin{array}{l}
E_{X} \\
E_{Y}
\end{array}\right]=\frac{\sqrt{2}}{4} E_{i n}\left\{\begin{array}{l}
{\left[\begin{array}{l}
(j+1) J_{0}(m)+(j-1) J_{1}(m) \exp \left(j \omega_{R F} t+j \varphi_{1}+j \varphi_{2}\right) \\
-(j-1) J_{1}(m) \exp \left(-j \omega_{R F} t+j \varphi_{1}+j \varphi_{2}\right)
\end{array}\right]} \\
{\left[\begin{array}{l}
J_{0}(m)+J_{1}(m) \exp \left(j \omega_{R F} t+j \varphi_{1}+j \varphi_{2}\right) \\
-J_{1}(m) \exp \left(-j \omega_{R F} t+j \varphi_{1}+j \varphi_{2}\right)+1
\end{array}\right]}
\end{array}\right\}
$$

where, $\varphi_{1}=\beta_{21} \omega_{\mathrm{RF}}^{2} L_{1} / 2, \varphi_{2}=\beta_{22} \omega_{\mathrm{RF}}^{2} L_{2} / 2, \quad \beta_{21}=\lambda^{2} D_{1} / 2 \pi c, \quad \beta_{22}=-\lambda^{2} D_{2} / 2 \pi c, \quad \beta_{21}$ is the second-order dispersion coefficient of the uplink SMF, whose length is $L_{1}$. $\beta_{22}$ is the second-order dispersion coefficient of DCF, whose length is $L_{2} . D_{1}$ and $D_{2}$ are total fiber dispersion of SMF and DCF, respectively. $\lambda$ denotes the wavelength of the optical carrier and $c$ is the velocity of light in vacuum.

After the DCF, the output optical signal is controlled by the PC1 to align the polarization states to the two principal axes of the PBS. Then the optical is polarization-demultiplexed into 
two paths. They are injected to PDx and PDy, respectively. Assuming $R_{i}(i=1,2)$ is the responsivity of the PDs. Exclude the direct current (DC) component through a DC block, the detected electrical power can be expressed as

$$
\begin{gathered}
P_{\text {upper }}(t)=R_{1} J_{0}^{2}(m) J_{1}^{2}(m) \cos ^{2}\left(-\frac{1}{2} \omega_{R F}^{2}\left(\beta_{21} L_{1}+\beta_{22} L_{2}\right)\right) \\
=R_{1} J_{0}^{2}(m) J_{1}^{2}(m) \cos ^{2}\left(\frac{\pi \lambda^{2} f^{2}\left(D_{1} L_{1}+D_{2} L_{2}\right)}{c}\right) \\
P_{\text {lower }}(t)=\frac{1}{4} R_{2}\left(J_{0}(m)+1\right)^{2} J_{1}^{2}(m) \sin ^{2}\left(-\frac{1}{2} \omega_{R F}^{2}\left(\beta_{21} L_{1}+\beta_{22} L_{2}\right)\right) \\
=\frac{1}{4} R_{2}\left(J_{0}(m)+1\right)^{2} J_{1}^{2}(m) \sin ^{2}\left(\frac{\pi \lambda^{2} f^{2}\left(D_{1} L_{1}+D_{2} L_{2}\right)}{c}\right)
\end{gathered}
$$

Based on Eqs. (3) and (4), an amplitude comparison function (ACF) can be constructed. It should be noted that when the driven signal power is sufficiently low, $J_{0}(m) \approx 1$. Therefore, ACF that defined as the power ratio between the two detected powers is given by

$$
A C F=\frac{P_{\text {lower }}}{P_{\text {upper }}}=K \frac{\sin ^{2}\left(\frac{\pi \lambda^{2} f^{2}\left(D_{1} L_{1}+D_{2} L_{2}\right)}{c}\right)}{\cos ^{2}\left(\frac{\pi \lambda^{2} f^{2}\left(D_{1} L_{1}+D_{2} L_{2}\right)}{c}\right)}=K \tan ^{2}\left(\frac{\pi \lambda^{2} f^{2}\left(D_{1} L_{1}+D_{2} L_{2}\right)}{c}\right)
$$

where, $K$ is the power ratio caused by different amplitudes response in two branches. Eq. (5) shows that the ACF only depends on the unknown microwave frequency and the fiber dispersion coefficient, which is not related to the input optical power or the microwave power in theory. Fig. 2 shows the power responses versus different microwave frequencies of the two paths. The related ACF is also calculated and plotted in Fig. 2. Here, a peak frequency $f_{\text {peak }}$ is achieved. It can be seen that when the frequency range is lower than the frequency of first peak $\left(f_{\text {peak }}\right)$, the ACF curve is monotonous. It means that the unknown microwave frequency can be estimated if the frequency is lower than the $f_{\text {peak }}$. The peak frequency is given as

$$
f_{\text {peak }}=\sqrt{\frac{c}{2 \lambda^{2}\left(D_{1} L_{1}+D_{2} L_{2}\right)}}
$$

Based on the theoretical analysis in Eq. (5), a significant problem should be figured out. When the location of the RAU is confirmed, the SMF that connects the RAU and the CS is also fixed, which means the fiber length $L_{1}$ and the dispersion coefficient $D_{1}$ are fixed. In this occasion, the related frequency measurement range is also fixed. It is not suitable for the practical circumstance. In practical, a RAU usually requires the ability of multi-band frequency measurement. If the frequency measurement range is fixed, the RAU can only work in a special band. Fortunately, as can be seen from Eq. (6), $f_{\text {peak }}$ not only depends on two parameters, $D_{1}$, and $L_{1}$, but also $D_{2}$, and $L_{2}$. It means, properly adjust the parameters such as $D_{2}$, and $L_{2}$ of the DCF in the CS, the frequency measurement range can also be tunable, which makes the remote frequency measurement system more flexible. 


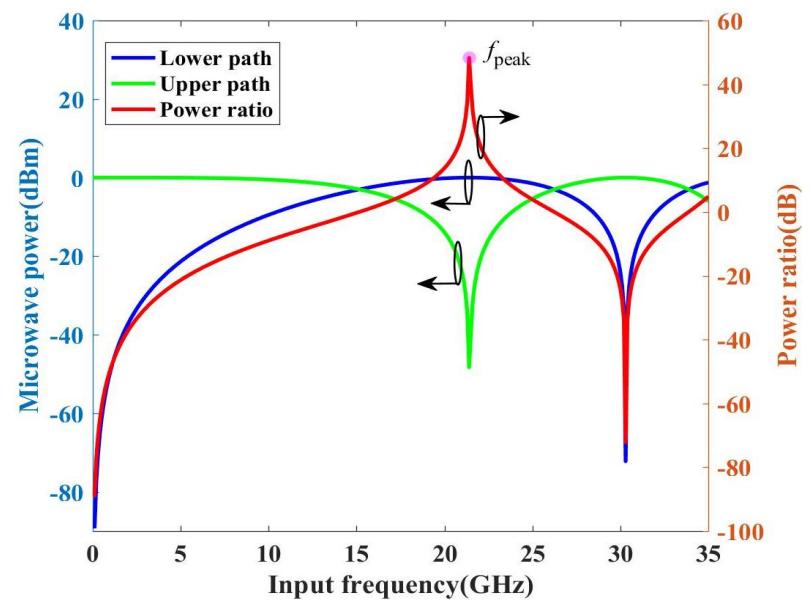

Fig. 2.Power fading characteristics of the two paths and the related ACF.

According to Eq. (5), ACF response can be calculated. First, the condition of no DCF in the CS is considered. Setting the main parameters of $\lambda=1550 \mathrm{~nm}, D_{1}=17 \mathrm{ps} / \mathrm{nm} / \mathrm{km}$ and changing the SMF length of $L_{1}=2.1 \mathrm{~km}$ to $4.1 \mathrm{~km}, 6.1 \mathrm{~km}, 8.1 \mathrm{~km}$, and $10.1 \mathrm{~km}$, the simulated curves are shown in Fig. 3. It can be seen that the related first peak frequency is $41.82 \mathrm{GHz}, 29.93 \mathrm{GHz}$, 24.53 GHz, $21.29 \mathrm{GHz}$, and $19.07 \mathrm{GHz}$ as the SMF length varies.

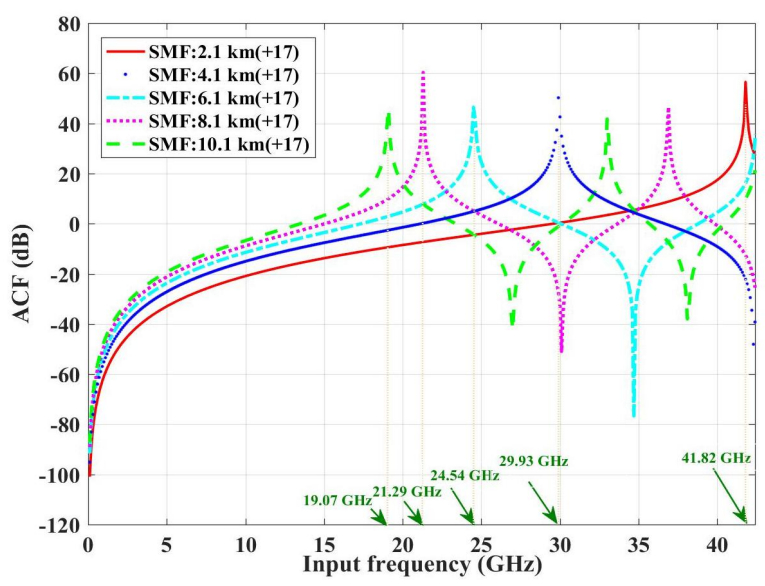

Fig. 3. Calculated ACF for different SMF lengths.

Then, focus on the measurement results in the case of DCF-assisting. Choosing two RAUs called $\mathrm{RAU}_{1}, \mathrm{RAU}_{2}$ with the distances of $8.1 \mathrm{~km}$ and $10.1 \mathrm{~km}$ as the examples. Both of them add the DCFs $\left(D_{2}=-160 \mathrm{ps} / \mathrm{nm} / \mathrm{km}\right)$ with the lengths of $0.5 \mathrm{~km}$, respectively. The calculated results are shown in Fig. 4, when compared to the results in Fig. 3, it can be shown that the maximum measurement point of the SMF with the $8.1 \mathrm{~km}$ is moved to $32.90 \mathrm{GHz}$. Similarly, the maximum measurement point of the SMF with the length of $10.1 \mathrm{~km}$ is expanded to 26.09 GHz. It means that DCF-assisting in the CS can flexibly control the measurement range. For different RAUs located at different places, the related length of SMF is also different. It means that the maximum measurement point would be varied without DCF. As for this problem, the DCF can also uniform the measurement ranges of the RAUs that locate in different places. As shown in Fig. 4, the ACF responses of the red curve $\left(R A U_{1}\right)$ and green curve $\left(R^{2} U_{2}\right)$ are different. However, when the DCF length of the $\mathrm{RAU}_{2}$ is changed to $0.7125 \mathrm{~km}$, the related ACF response (blue dot curve) is the same as that of RAU ${ }_{1}$. Similarly, focus on the RAU $U_{1}$, 
change the length and the dispersion coefficient of the DCF to $0.23 \mathrm{~km}$ and $-200 \mathrm{ps} / \mathrm{nm} / \mathrm{km}$, respectively. The ACF response is also equal to that of $\mathrm{RAU}_{2}$.

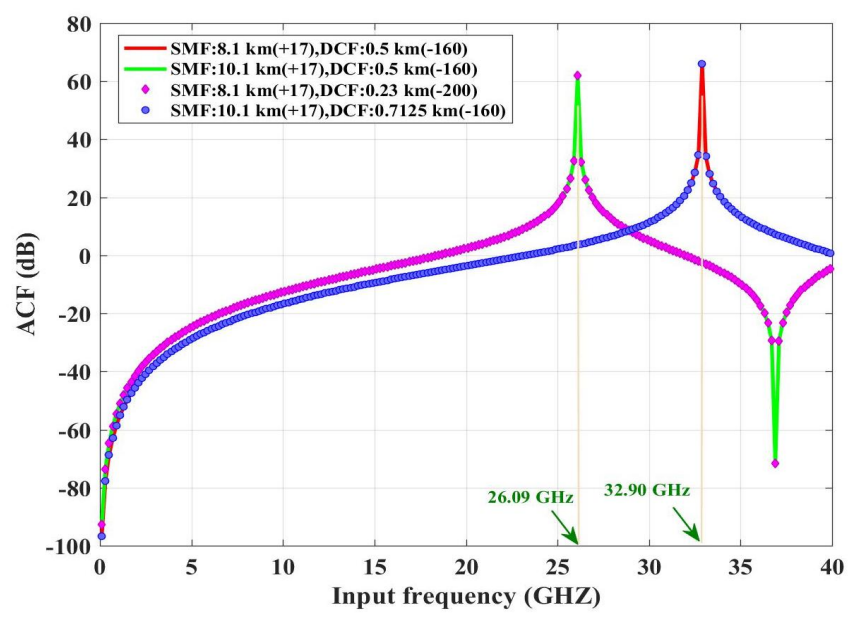

Fig. 4. Calculated ACF for different DCF-assisting.

\section{Simulation and discussion}

To investigate the validity of this methodology, simulations are performed based on the setup in Fig. 1(b) using OptiSystem 14.0. A light carrier at $1550 \mathrm{~nm}$ with a linewidth of 0.5 $\mathrm{MHz}$ generated from a CW laser is equally splitted and injected into the DPol-DMZM. It is modulated by a microwave signal, whose frequency is tuned from $5 \mathrm{GHz}$ to $35 \mathrm{GHz}$ with a step of $500 \mathrm{MHz}$, and the bias state of the DMZM1 is maintained at quadrature point ( $V_{\text {bias }}=$ $V_{\pi} / 2$ ).Here the chromatic dispersion value of SMF is set at $17 \mathrm{ps} / \mathrm{nm} / \mathrm{km}$ and the length is 8.1 $\mathrm{km}$. The ACF is calculated by the measured power of the upper and lower branches. As the results shown in Fig. 5(a), we can see that the measured ACF is in good agreement with the theoretical value, and their measurement range is $4.75-21.25 \mathrm{GHz}$. Based on the measured value of ACF, the unknown microwave RF signal frequency can be deduced and the accuracy of the measured frequency is given in Fig. 5(b). It can be seen that in the range of 4.75-21.25 $\mathrm{GHz}$, the measurement error is better than $\pm 0.2 \mathrm{GHz}$.

In order to research the ability of measurement range controlling, the length and the chromatic dispersion value of SMF is set to be $10.1 \mathrm{~km}$ and $17 \mathrm{ps} / \mathrm{nm} / \mathrm{km}$, respectively. The chromatic dispersion value of DCF is fixed at $-160 \mathrm{ps} / \mathrm{nm} / \mathrm{km}$. $L_{2}$ is tuned to be $0 \mathrm{~km}, 0.2125$ $\mathrm{km}$ and $0.7125 \mathrm{~km}$ and the peak measurement point is tunable from $19.07 \mathrm{GHz}$ to $21.25 \mathrm{GHz}$ and $32.90 \mathrm{GHz}$, as shown in Fig. 6(a). It can be concluded that DCF changes the maximum measurement range and enhances the performance of the system, which can realize adjustable microwave frequency measurement. Compared to the red star curve of Fig. 6(a) and the red diamond curve of Fig. 5(a), we can find that through controlling the length or the chromatic dispersion value of DCF, a SMF with the length of $10.1 \mathrm{~km}$ shows the same $f_{\text {peak }}(21.25 \mathrm{GHz})$ to that of the SMF with the length of $8.1 \mathrm{~km}$. This means that if the parameters of DCF in the CS are properly adjusted, different RAUs can work in the same measurement range without changing the length of SMF. Then, we further examine the errors between the simulated frequency and the theoretical value with the peak measurement point of $32.90 \mathrm{GHz}$ as shown in Fig. 6(b). The measurement range is $8-32.75 \mathrm{GHz}$ with a measurement accuracy of \pm 0.2 GHz. Higher frequency measurement can still be realized by simply adjusting the length of 
DCF in CS. The lower frequency measurement can be easily achieved using the conventional electrical IFM approaches [3, 4].
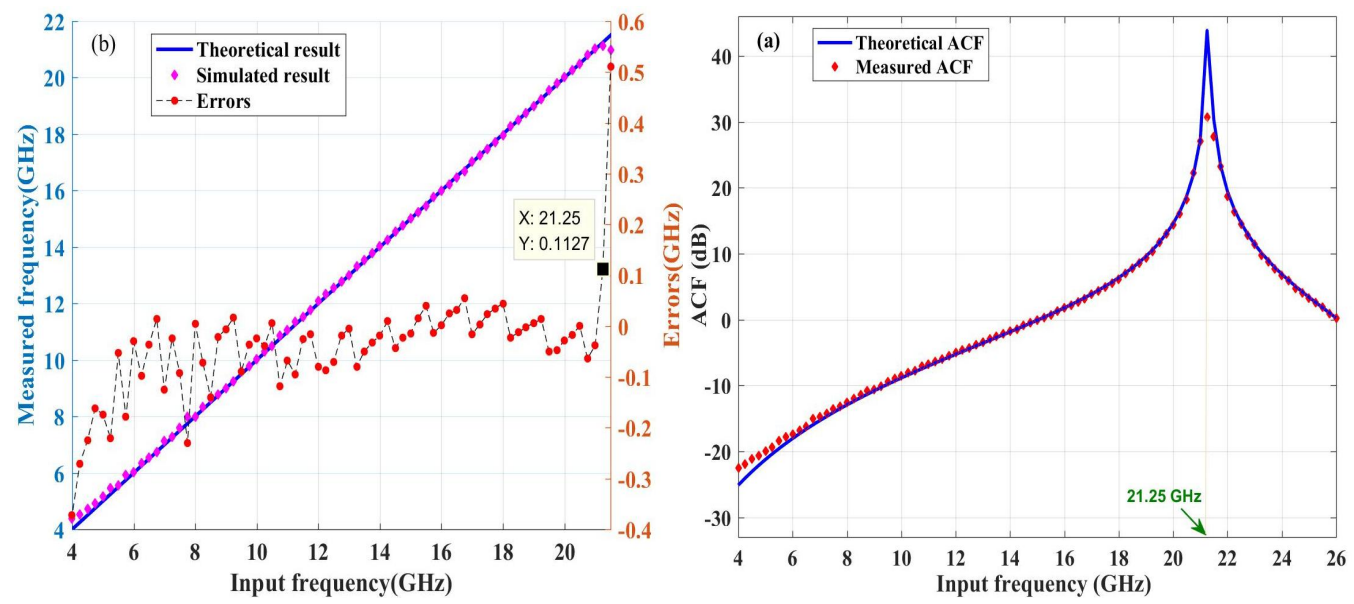

Fig. 5. (a) Comparison of the calculated ACF and the simulated ACF as D1=17 ps/nm/km, L1=8.1 km. (b) Simulated microwave frequency versus theoretical result and the errors of the simulated frequency relative to the theoretical value as $\mathrm{L} 1=8.1 \mathrm{~km}$.
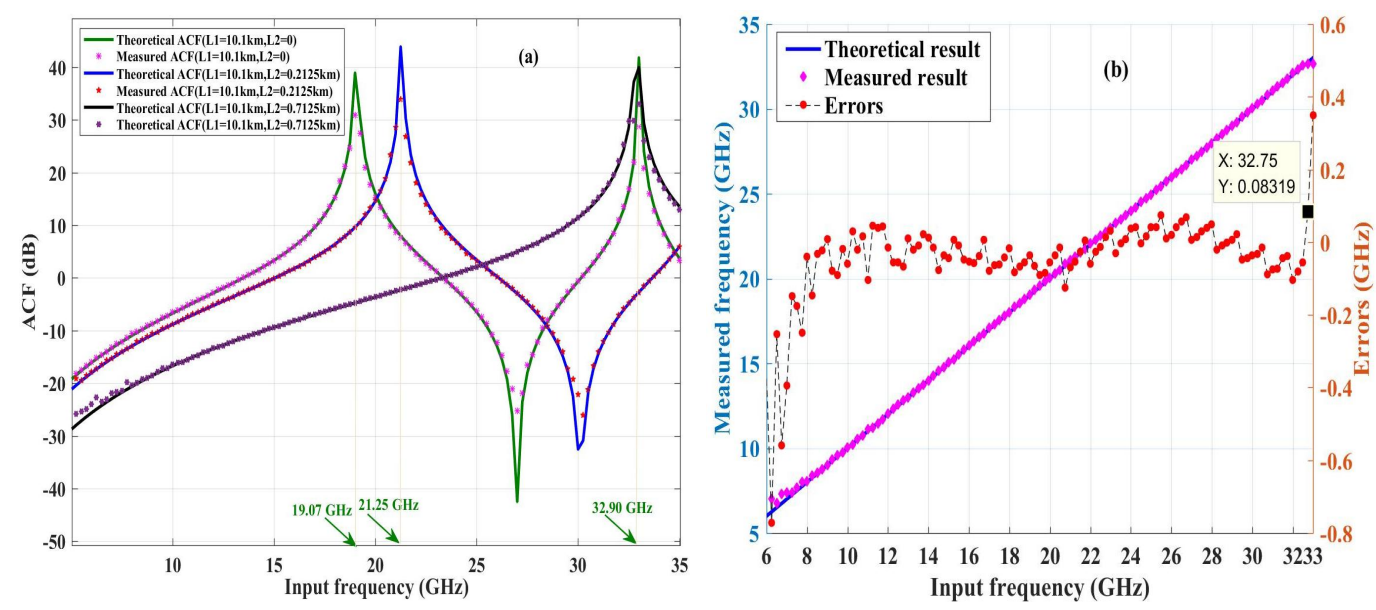

Fig. 6. (a) Comparison of the calculated ACF and the simulated ACF at $L_{1}=10.1 \mathrm{~km}$ with different length of $L_{2}$. (b) Simulated microwave frequency versus theoretical result and the errors of the simulated frequency relative to the theoretical value as $L_{2}=0.7125 \mathrm{~km}$.

Other factors that cause measurement errors such as DC drifting, intensity noise (IN), phase noise (PN), equivalent deviation of PBS and the dispersion value of the SMF are also considered and analyzed. Firstly, the DC bias voltage of DMZM may drift and affect the feature of the proposed RFM system. To evaluate it, the DC drifting is tuned from $-20 \%$ to $20 \%$ with a step of $10 \%$ while the other parameters are fixed. As is shown in Fig. 7(a), when the DC drifting is $20 \%$, the measurement errors are higher than $0.7 \mathrm{GHz}$ at low frequency range. This means the RFM would be influenced by the DC drifting. Fortunately, this problem can be well solved if the DC bias feedback controlling circuit is available.

In general, the linewidth of the LD is related to the IN and PN [18]. Thus, we can try to adjust the linewidth to investigate the influence of IN and PN. Fig. 7(b) shows the measurement results. It can be seen that as the linewidth increases, the measurement errors continue to increase in the lower frequency range. Fortunately, at the higher frequency range 
(18-34.25 GHz), this system has a good stability against the non-ideal characters of the LD. In fact, photonic-based RFM is investigated to solve the high frequency measurement problem that electrical method faces. Therefore, the stable performance of this RFM over the high frequency range shows a good prospect of large range frequency measurement.

Polarization multiplexing and de-multiplexing are the main technologies to guarantee good isolation between the intensity modulation and phase modulation. Usually, the PC is adjusted by hand to make sure that the polarization states are aligned to the principal axes. It is not an accurate operation. Therefore, whether the measurement error would be influenced by the non-aligned polarization states is worth considering. Fig.7(c) shows the measurement results under different polarization states deviation. Apparently, the non-aligned polarization states affect the measurement resolution. Noteworthy, the measurement error in the high frequency range still keeps in a stable level of lower than $\pm 0.2 \mathrm{GHz}$ as the polarization states deviate.

According to the theoretical results shown in Eq. (6), apart from the tuning of fiber length of the DCF, the dispersion coefficient can be also tuned to adjust the measurement range. Therefore, it is important to investigate the influence of the dispersion coefficient on the measurement error. Fig. 7(d) shows the measurement results at different dispersion coefficient of DCF. We can see that the instability of the dispersion coefficient has little effect on the measurement error over the high frequency range.
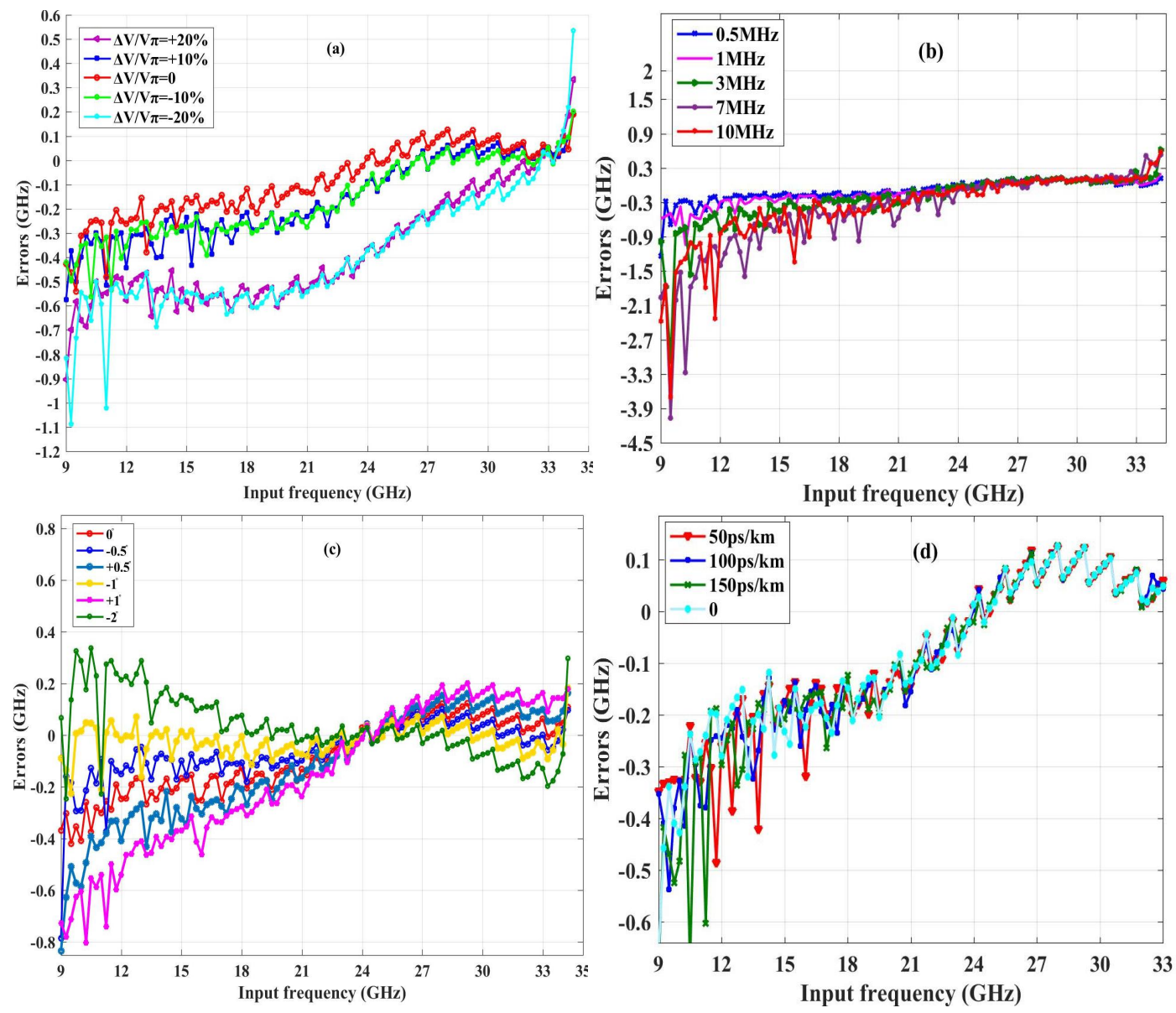

Fig. 7. (a) Simulated ACF at different DC drifting. (b) Simulated ACF at different linewidth of the LD. (c) Simulated ACF at different polarization states deviation of PC1. (d) Simulated ACF at different dispersion coefficient of DCF. 


\section{Conclusion}

We proposed a flexible RFM system with adjustable measurement range based on an optical fiber dispersion compensation method. By controlling the length or the chromatic dispersion value of the DCF, the measurement range of each RAU can be flexibly adjusted and different RAUs can be easily controlled to work at the same measurement range by simply adjust the parameters of the dispersion medium in CS. More importantly, the proposed novel system not only has a significantly simplified structure, but also effective in ensuring the concealment of the signal processing unit that set in CS. Some non-ideal factors such as the bias drift, intensity noise, phase noise, the equivalent deviation of PBS, and the dispersion value of the DCF that cause measurement errors are discussed. The simulation results shown that the non-ideal factors has little influence on the measurement performance for high frequency range.

\section{Disclosure statement}

No potential conflict of interest was reported by the authors.

\section{Acknowledgments}

This research was supported in part by the National Natural Science Foundation of China under Grant 61901507, Grant 62001505, in part by the Natural Science Foundation of Shaanxi Province under Grant Grant 2020JQ-469.

\section{References}

[1] Zhang B, Wang X, and Pan S. Photonics-based instantaneous multiparameter measurement of a linear frequency modulation microwave signal. J. Lightw. Technol. 2018; 36: 2589-2596.

[2] Zhu D and Pan S. Broadband cognitive radio enabled by photonics. J. Lightw. Technol. 2020; 38:3076-3088.

[3] Guo YT, Ma J X. Photonic instantaneous microwave frequency measurement with adjustable measurement range based on an electro-optical polarization modulator. OSA. Appl. Optics 2020;59: 1808-1816.

[4] Li J, Pei L, Ning TG,et al. Measurement of instantaneous microwave frequency by optical power monitoring based on polarization interference. J. Lightw. Technol. 2020; 38: 2285-2291. [5] Li Z, Zhang XM, Chi H, et al. A reconfigurable microwave photonic channelized receiver based on dense wavelength division muiltiplexing using an optical comb. Opt. Commun. 2012; 285:2311-2315.

[6] Wang C, Yao J. Ultrahigh-resolution photonic-assisted microwave frequency identification based on temporal channelization. IEEE Trans. Microw. Theory Techn. 2013; 61: 4275-4282.

[7] Ye C, Fu H, Zhu K, et al. All-optical approach to microwave frequency measurement with large spectral range and high accuracy. IEEE Photonics Technol. Lett. 2012; 24:7, 614-616.

[8] Hao T, Tang J, Li W, et al. Microwave photonics frequency-to-time mapping based on a fourier domain mode locked optoelectronic oscillator. Opt. Express 2018; 26: 33582-33591 .

[9] Jiang J, et al. Photonic-assisted microwave frequency measurement system based on a silicon ORR. Opt. Commun. 2017; 382: 366-370.

[10] Li W, Zhu NH, Wang LX. Brillouin-assisted microwave frequency measurement with adjustable measurement range and resolution. Opt. Lett. 2012; 37: 166-168. 
[11] Li XY, Wen AJ, Ma XM, et al. Photonic microwave frequency measurement with a tunable range based on a dual-polarization modulator. OSA. Appl. Optics 2016; 55: 8727-8731.

[12] Zhu ZH, Merklein M, Choi DY, et al. Highly sensitive, broadband microwave frequency identification using a chip-based Brillouin optoelectronic oscillator. Opt. Express 2019; 27: 12855-12868.

[13] Yang CW, Yu WQ, Liu JG. Reconfigurable instantaneous frequency measurement system based on a polarization multiplexing modulator. IEEE Photon. J. 2019 ;11: 5500611.

[14] Zhao ZY , Zhu K, Lu LY, et al. Instantaneous microwave frequency measurement using few-mode fiber-based microwave photonic filters. Opt. Exp. 2020; 28: 37353-37361.

[15] Garrett LD, Eiselt MH. Bi-directional single-fiber transmission. Opt. Trans., Switching, and Subsystems II 2005; 5625: 454-461.

[16] Li S, Zheng X, Zhang H, et al. Compensation of dispersioninduced power fading for highly linear radio-over-fiber link using carrier phase-shifted double sideband modulation. Opt. Lett. 2011; 36:546-548.

[17] Tu ZY, Wen AJ, Gao YS, et al. A photonic technique for instantaneous microwave frequency measurement utilizing a phase modulator. IEEE Photon. Technol. Lett. 2016; 28: 2795-2798.

[18] Osinski M, Buus J . Linewidth broadening factor in semiconductor lasers-an overview. IEEE J. Quantum Electron. 1987; QE-23: 9-29. 